\title{
Non-aqueous capillary electrophoretic enantioseparation of $N$-derivatized amino acids using cinchona alkaloids and derivatives as chiral counter-ions
}

\author{
Véronique Piette $^{\mathrm{a}}$, Marianne Fillet ${ }^{\mathrm{b}}$, Wolfgang Lindner ${ }^{\mathrm{c}}$, Jacques Crommen ${ }^{\mathrm{b}, *}$ \\ ${ }^{a}$ Scientific Institute of Public Health - Louis Pasteur, rue Juliette Wytsman 14, B-1050 Bruxelle, Belgium \\ ${ }^{\mathrm{b}}$ Department of Analytical Pharmaceutical Chemistry, Institute of Pharmacy, University of Liège, CHU, B36, B-4000 Liège 1, Belgium \\ ${ }^{\mathrm{c}}$ Institute of Analytical Chemistry, University of Vienna, Währingerstrasse 38, A-1090 Vienna, Austria
}

\begin{abstract}
A non-aqueous capillary electrophoretic method developed with quinine and tert-butyl carbamoylated quinine as chiral selectors for the enantioseparation of $N$-protected amino acids was applied to the investigation of other quinine derivatives as chiral additives. The optimum composition of the background electrolyte was found to be $12.5 \mathrm{~m} M$ ammonia, $100 \mathrm{~m} M$ octanoic acid and $10 \mathrm{~m} M$ chiral selector in an ethanol-methanol (60:40, v/v) mixture. Under these conditions, a series of chiral acids, as various benzoyl, 3,5-dinitrobenzoyl and 3,5-dinitrobenzyloxycarbonyl amino acid derivatives were investigated with regards to selectand-selector relationships and enantioselectivity employing quinine, quinidine, cinchonine, cinchonidine, tert.-butyl carbamoylated quinine, tert.-butyl carbamoylated quinidine, dinitrophenyl carbamoylated quinine and cyclohexyl carbamoylated quinine as chiral selector. (c) 2000 Elsevier Science B.V. All rights reserved.
\end{abstract}

Keywords: Non-aqueous capillary electrophoresis; Enantiomer separation; Chiral selectors; Buffer composition; Amino acids; Alkaloids

\section{Introduction}

In capillary electrophoresis (CE), enantiomeric separations are most often obtained by addition of the chiral selector ( $\mathrm{SO}$ ) to the background electrolyte [1-4]. Unfortunately, some of these chiral selectors, used successfully on stationary phases in chiral highperformance liquid chromatography (HPLC) have only limited solubility in aqueous buffers which are generally used in CE. Non-aqueous CE (NACE), employing small amounts of well-characterized chiral additives, offers an attractive alternative for

\footnotetext{
*Corresponding author. Tel.: +32-4-3664-346; fax: +32-4-3664347.

E-mail address: jcrommen@ulg.ac.be (J. Crommen)
}

screening the chiral discrimination potential of selectors with low solubility in water and studying the interactions between these selectors and various kinds of analytes [5,6].

Quinine has been used as a chiral solvating agent in nuclear magnetic resonance (NMR) studies [7], as a chiral ion-pairing agent for enantioseparation in HPLC $[8,9]$ as well as in capillary electrochromatography (CEC) [10] and as a selector immobilized on a chiral stationary phase in HPLC $[11,12]$. Chiral stationary phases based on the use of carbamoylated derivatives of quinine and quinidine as selectors were found to be highly stereoselective for the direct resolution of chiral acids in HPLC using mixtures of aqueous buffers and methanol or acetonitrile as mobile phases [13-18]. This new class of chiral 
selectors can be classified as weak anion exchangers, due to the presence of a tertiary amino group within the quinuclidine ring which is protonated at the usual working $\mathrm{pH}$ of the mobile phase. The primary ionic interaction between the anionic solutes (selectands, SAs) and the cationic SO is significantly accompanied by additional intermolecular interactions as hydrogen bonding, dipole-dipole, charge transfer $(\pi-\pi)$, hydrophobic and steric interactions. These simultaneously acting multiple interactions can be seen as a basis for the high stereoselectivity potential shown by these SOs. Moreover, the introduction of a bulky alkyl substituent at the carbamate function turned out to be advantageous for a further increase in stereoselectivity $[13,14]$.

Recently, a NACE system using a background electrolyte composed of $12.5 \mathrm{~m} M$ of ammonium acetate in methanol was found to be useful for the investigation of the potential of quinine and tert.butyl carbamoylated quinine as chiral SOs for the
(1)

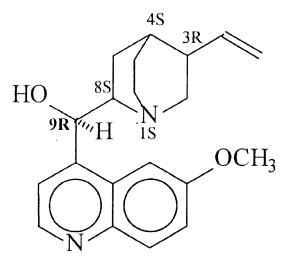

(3)

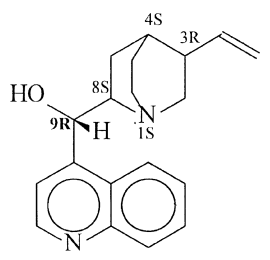

(5)

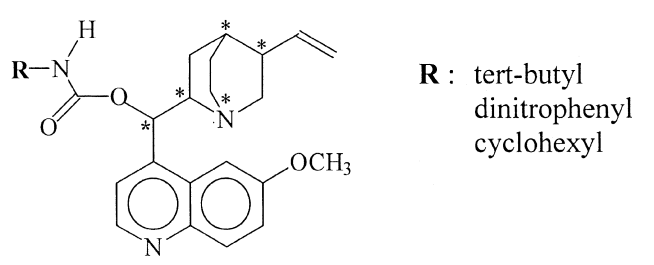

Fig. 1. Chiral selectors. (1) Quinine, (2) quinidine, (3) cinchonine, (4) cinchonidine and (5) carbamoylated quinine derivatives.
(1)

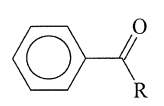

(2)

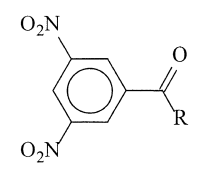

(3)

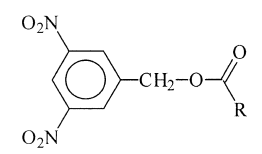

R : Leucine (Leu)

Phenylglycine (Phg)

$\alpha$-Methyl-Leucine $(\alpha$ MeLeu)

$\mathrm{N}$-Methyl-Leucine (NMeLeu)

tert-Leucine (tLeu)

Phenylalanine (Phe)

$\beta$-Phenylalanine ( $\beta$ Phe)

Proline (Pro)

$\alpha$-amino-butyric acid ( $\alpha \mathrm{Abu})$

$\beta$-amino-butyric acid ( $\beta$ Abu)

pipecolinic acid (PA)
Fig. 2. $N$-Protected amino acid derivatives. (1) Bz (benzoyl), (2) DNB (3,5-dinitrobenzoyl), (3) DNZ (3,5-dinitrobenzyloxycarbonyl).

enantioseparation of $\mathrm{N}$-protected amino acids [19]. The influence of the nature of the organic solvent, the combination of different solvents, the nature and the concentration of the background electrolyte, the concentration of the selector, the capillary temperature and the applied voltage was systematically studied, using DNB-Leu and DNB-Phg as model compounds. A buffer made of $12.5 \mathrm{~m} M$ ammonia, $100 \mathrm{~m} M$ octanoic acid and $10 \mathrm{~m} M$ SO in an ethanolmethanol (60:40) mixture was found to give the best compromise in terms of selectivity, resolution, efficiency, peak symmetry and analysis time [19].

In this study, such a NACE system was applied for the rapid screening of different kinds of cinchona alkaloids and derivatives tested as SOs: quinine $(\mathrm{QN})$, quinidine (QD), cinchonine $(\mathrm{CN})$, cinchonidine (CD), tert.-butyl carbamoylated quinine (tBuCQN), tert.-butyl carbamoylated quinidine (tBuCQD), dinitrophenyl carbamoylated quinine (DNPCQN) and cyclohexyl carbamoylated quinine (cHexCQN) (cf. Fig. 1). A series of various $\mathrm{N}$ protected amino acid derivatives were tested as chiral acidic selectands, including Bz- (benzoyl), DNB(3,5-dinitrobenzoyl) and DNZ- (3,5-dinitrobenzyloxycarbonyl) amino acids (cf. Fig. 2).

\section{Experimental}

\subsection{Apparatus}

All experiments were performed on a Spec- 
traphoresis $1000 \mathrm{CE}$ instrument (Spectraphysics, San Jose, CA, USA) equipped with an autosampler, a UV-visible detector $(190-800 \mathrm{~nm})$ and a temperature control system $\left(15-60^{\circ} \mathrm{C}\right)$. An IBM PS $/ 2$ Model 90486 was used for instrument control and data handling. Electropherograms were printed on a HP DeskJet 500 printer. A column cartridge was obtained from Spectraphysics. The apparent $\mathrm{pH}\left(\mathrm{pH}_{\mathrm{a}}\right)$ of the non-aqueous electrolyte solutions was measured by means of a Model Delta $345 \mathrm{pH}$ meter from Mettler (Healstead, UK).

\subsection{Chemicals and reagents}

QN was obtained from Sigma (St. Louis, MO, USA); $\mathrm{QD}, \mathrm{CN}$ and $\mathrm{CD}$ were from Buchler (Brannschweig, Germany). TBuCQN, tBuCQD, DNPCQN and cHexCQN were synthesized according to a standard procedure described elsewhere [20]. The organic solvents were HPLC-grade: ethanol absolute from Merck (Darmstadt, Germany) and methanol from Fisher Scientific (Leicestershire, UK). Octanoic acid was from Sigma and ammonia solution 25\% from Carlo Erba (Rodano, Italy). The amount of water in electrophoresis media was determined by Karl-Fisher titration using Hydranal Composit 1 from Riedel-de Häen (Seelze, Germany) standardized against sodium tartrate dihydrate (15.66\% water, Merck). The racemic and enantiomerically pure amino acids were purchased from Sigma. Bz and DNB derivatives were synthesized according to standard derivatization procedures [17] except DNB-Leu and DNB-Phg obtained from Sigma. To synthesize the DNZ compounds, aqueous solutions of amino acid were derivatized with 3,5dinitrobenzyl chloroformate [18]. The sample solutions were prepared by dissolving each amino acid derivative at a concentration of $50 \mu \mathrm{g} / \mathrm{ml}$ in methanol. Benzylic alcohol from Sigma $(0.01 \%$ methanolic solution) was used as neutral marker to visualize the electroosmotic flow $\mu_{\mathrm{EOF}}$. Buffers and samples were filtered through a Polypure polypropylene membrane filter $(0.2 \mu \mathrm{m})$ from Alltech (Laarne, Belgium) before use.

\subsection{Electrophoretic technique}

Electrophoretic separations were carried out with uncoated fused-silica capillaries, $44 \mathrm{~cm}(37 \mathrm{~cm}$ to the detector) $\times 50 \mu \mathrm{m}$ I.D., provided by Supelco (Bellefonte, PA, USA). The buffer was made of $100 \mathrm{~m} M$ octanoic acid and $12.5 \mathrm{~m} M$ ammonia in a mixture of ethanol-methanol (60:40). The amount of water determined in this electrophoretic medium was $0.25 \%$. At the beginning of each working day, the capillary was washed with ethanol-methanol (60:40) for $5 \mathrm{~min}$ and with the running buffer for $10 \mathrm{~min}$, while after each injection the capillary was washed with the solvent mixture for $1 \mathrm{~min}$ and was equilibrated with the buffer mixture for $10 \mathrm{~min}$. The injections were made at the cathodic side and the applied voltage was $-25 \mathrm{kV}$ (reversed polarity mode). The normal polarity mode $(+25 \mathrm{kV})$ was used to measure the cathodic electroosmotic flow $\mu_{\text {EOF }}$ (current: about $6.5 \mu \mathrm{A}$ ). The separations were performed with the electrolyte solution containing the selector in the reservoir at the cathodic side and with the same electrolyte solution devoid of the selector in the reservoir at the anodic side. The UV detection (at the anodic side) was performed at 214 $\mathrm{nm}$. Injections were made in the hydrodynamic mode for a period of $5 \mathrm{~s}$ (corresponding to $13.3 \mathrm{nl}$ ) and the capillary was thermostated at $15^{\circ} \mathrm{C}$. The resolution $\left(R_{s}\right)$ and the plate number $(N)$ were calculated according to the standard expressions based on peak width at half-height [21]. The asymmetry factor $\left(A_{\mathrm{s}}\right)$ was determined using the expression: $A_{\mathrm{s}}=w(0.10) /$ $2 F(0.10)$ where $w(0.10)$ is the width of peak at $10 \%$ of height and $F(0.10)$ is the distance between the front edge and the top of the peak at $10 \%$ of peak height. The selectivity $(\alpha)$ was calculated according to $\alpha=\mu_{\mathrm{e} 1} / \mu_{\mathrm{e} 2}$ where $\mu_{\mathrm{e}}=\mu_{\mathrm{a}}-\mu_{\mathrm{EOF}}$ ( $\mu_{\mathrm{e}}$ is the effective mobility, $\mu_{\mathrm{a}}$ is the apparent mobility and $\mu_{\mathrm{EOF}}$ is the electroosmotic mobility).

\section{Results and discussion}

\subsection{Detection of the analytes in the presence of a highly UV absorbing selector}

Cinchona alkaloids and their derivatives exhibit high molar absorptivities at the detection wavelength used $(214 \mathrm{~nm})$, so that they might give rise to a high absorbance background when they are added to the background electrolyte, making the direct UV detection of the analyte enantiomers difficult.

In order to cope with this problem, the electrolyte 
solution introduced in the reservoir at the anodic (detector) side was devoid of the UV absorbing chiral selector [19].

Due to the electrophoretic migration of the positively charged selector towards the cathode (injection side) and the presence of a cathodic electroosmotic flow, the part of the capillary situated close to the detection window was progressively depleted from the chiral selector during the run and after a certain time (6-12 min after the injection, depending of the nature of the chiral selector), a rapid decrease of the background absorbance was observed, indicating that the chiral selector had left the detection window (cf. Fig. 3). Since the migration times of the analyte enantiomers were generally higher than $13 \mathrm{~min}$, they could be detected with high sensitivity, in a portion of selector-free background electrolyte. The breakthrough times, corresponding to the disappearance of the selector from the detection window, were fairly reproducible for a given selector under the same operational conditions. Breakthrough times were somewhat lower, however, for cinchona alkaloids (QN: $7.7 \mathrm{~min}, \mathrm{QD}: 7.9 \mathrm{~min}, \mathrm{CN}$ : $5.6 \mathrm{~min}$ and $\mathrm{CD}$ : $6.5 \mathrm{~min})$ than for the carbamoylated derivatives (tBuCQN: 7.3 min, tBuCQD: 7.4 min, DNPCQN:
$11.8 \mathrm{~min}$ and CHexCQN: $8.6 \mathrm{~min}$ ) under the same conditions, which might indicate that the latter (DNPCQN in particular) have a more pronounced tendency to interact with the capillary wall. This is confirmed to some extent by the corresponding $\mu_{\mathrm{EOF}}$ values (in $10^{-5} \mathrm{~cm}^{2} / \mathrm{V} \cdot \mathrm{s}$ ) obtained with the different selectors. (QN: 2.4, QD: 2.3, CN: 3.0, CD: 2.8, tBuCQN: 2.2, tBuCQD: 2.3, DNPCQN: 1.5 and CHexCQN: 1.8).

\subsection{Selection of the experimental conditions for non-aqueous capillary electrophoresis}

In previous work [19], different background electrolytes were investigated for the optimization of the NACE method, using DNB-Leu and DNB-Phg as model compounds. A long chain buffer anion which present an electrophoretic mobility similar to that of the SAs was found to improve peak symmetry and efficiency.

The addition of ethanol to methanol gave rise to higher selectivity and resolution values. This solvent with hydrogen bonding properties may provide a better environment for achieving ion-pair interactions in contrast to addition of acetonitrile. The concen-

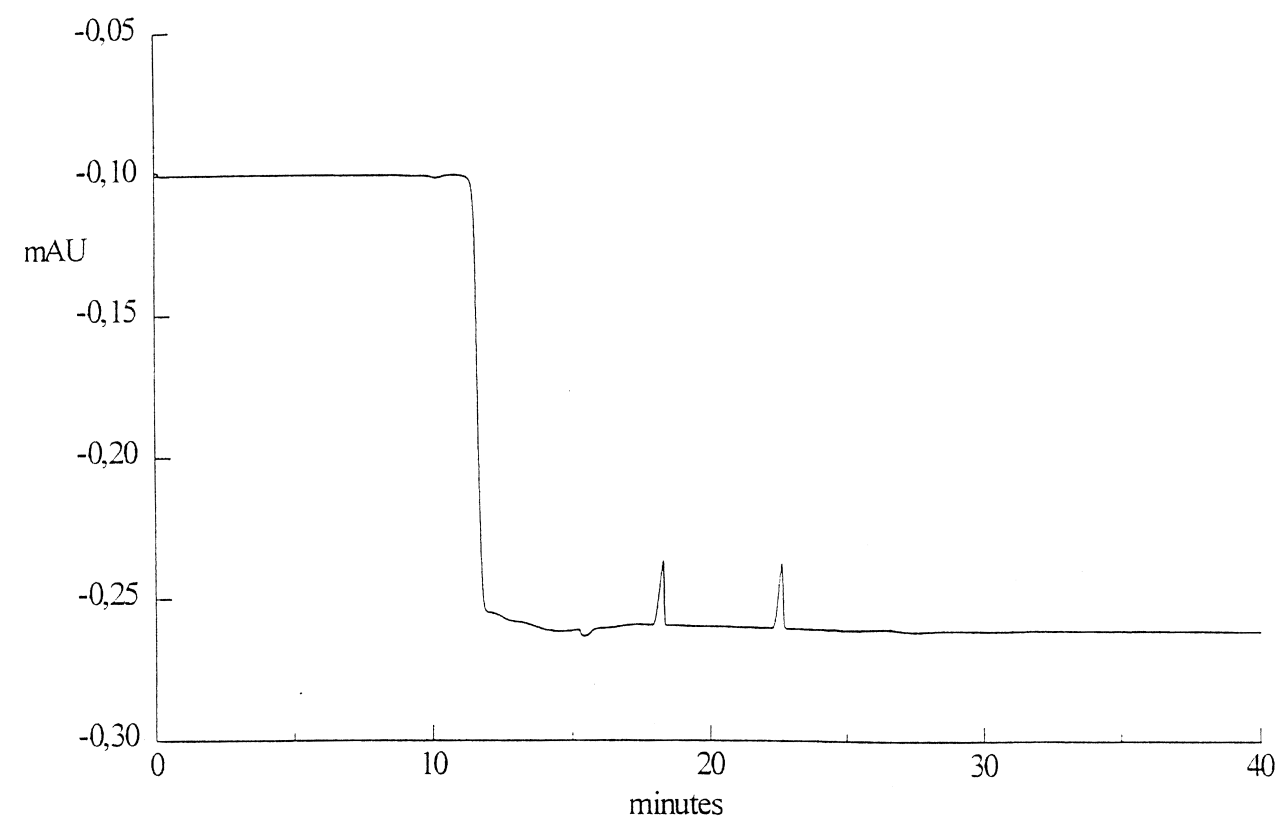

Fig. 3. Enantioseparation of DNB-Phe. Buffer: $100 \mathrm{~m} M$ octanoic acid and $12.5 \mathrm{~m} M$ ammonia in methanol-ethanol (40:60) containing 10 $\mathrm{m} M$ DNPCQN. 


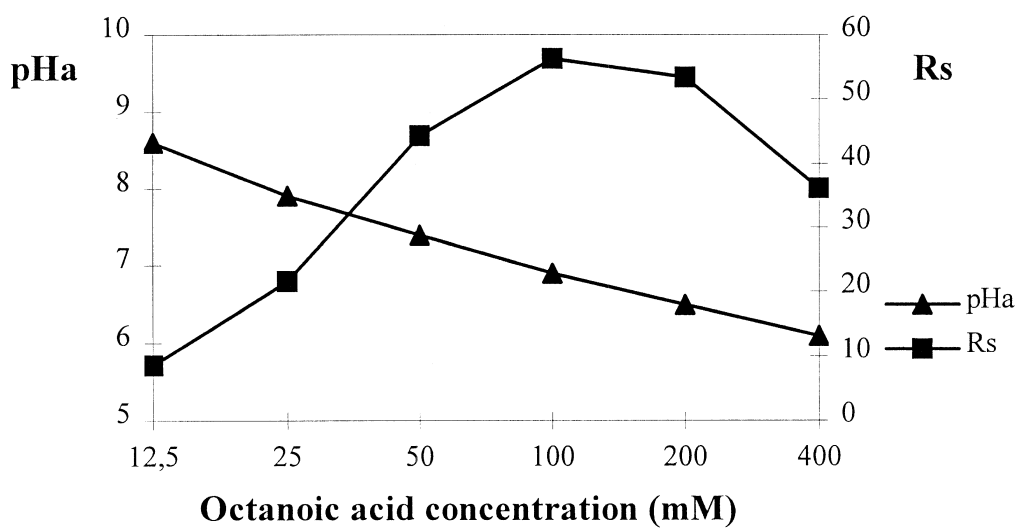

Fig. 4. Influence of the octanoic acid concentration on the $\mathrm{pH}_{\mathrm{a}}$ and on the resolution $\left(R_{\mathrm{s}}\right)$ of the DNB-Leu enantiomers. Buffer: $12.5 \mathrm{~m} M$ ammonia and $5 \mathrm{~m} M$ tBuCQN in methanol-ethanol (40:60). Other conditions as described in Section 2.3.

Table 1

Enantioresolution of amino acid derivatives with QN, QD, CN and CD as selectors ${ }^{\mathrm{a}}$

\begin{tabular}{|c|c|c|c|c|c|c|c|c|c|c|c|c|c|c|c|c|}
\hline \multirow[t]{2}{*}{ Analyte } & \multicolumn{4}{|l|}{ QN } & \multicolumn{4}{|l|}{ QD } & \multicolumn{4}{|l|}{$\mathrm{CN}$} & \multicolumn{4}{|l|}{$\mathrm{CD}$} \\
\hline & $\begin{array}{l}t_{1} \\
(\mathrm{~min})\end{array}$ & $\begin{array}{l}t_{2} \\
(\min )\end{array}$ & $\alpha$ & $R_{s}$ & $\begin{array}{l}t_{1} \\
(\min )\end{array}$ & $\begin{array}{l}t_{2} \\
(\min )\end{array}$ & $\alpha$ & $R_{s}$ & $\begin{array}{l}t_{1} \\
(\mathrm{~min})\end{array}$ & $\begin{array}{l}t_{2} \\
(\min )\end{array}$ & $\alpha$ & $R_{s}$ & $\begin{array}{l}t_{1} \\
(\mathrm{~min})\end{array}$ & $\begin{array}{l}t_{2} \\
(\min )\end{array}$ & $\alpha$ & $R_{s}$ \\
\hline DNB-Leu $^{\mathrm{b}}$ & 20.03 & 21.55 & 1.057 & 5.5 & 22.52 & 24.80 & 1.060 & 8.3 & 20.12 & 21.75 & 1.055 & 7.0 & 21.59 & 23.06 & 1.046 & 5.3 \\
\hline DNB-Phg & 15.73 & 16.54 & 1.041 & 4.3 & 14.08 & 15.04 & 1.048 & 6.3 & 16.25 & 17.07 & 1.037 & 4.8 & 23.16 & 24.16 & 1.028 & 3.7 \\
\hline DNB-tLeu & 19.17 & 19.61 & 1.018 & 2 & 13.90 & 14.26 & 1.019 & 2.3 & 18.96 & 19.49 & 1.020 & 2.6 & 18.59 & 18.97 & 1.014 & 1.8 \\
\hline DNB- $\alpha$ MeLeu & 16.40 & $-^{\mathrm{c}}$ & - & - & 20.14 & - & - & - & 15.51 & - & - & - & 16.51 & - & - & - \\
\hline DNB-NMeLeu & 15.60 & 15.81 & 1.011 & $<0.7$ & 21.46 & 21.75 & 1.008 & $<0.7$ & 16.23 & 16.42 & 1.009 & $<0.7$ & 17.78 & 17.94 & 1.006 & $<0.7$ \\
\hline DNB-Phe ${ }^{b}$ & 19.90 & 20.55 & 1.025 & 2.6 & 14.93 & 15.54 & 1.028 & 3.6 & 19.08 & 19.82 & 1.027 & 3.1 & 22.34 & 23.07 & 1.022 & 2.7 \\
\hline DNB-Pro & 14.40 & - & - & - & 15.76 & - & - & - & 18.83 & - & - & - & 21.13 & - & - & - \\
\hline DNB- $\alpha \mathrm{Abu}$ & 18.77 & 19.92 & 1.047 & 4.8 & 14.77 & 16.07 & 1.060 & 7.6 & 19.28 & 20.47 & 1.043 & 5.6 & 18.72 & 19.69 & 1.036 & 4.1 \\
\hline DNB- $\beta$ Abu & 35.04 & 38.13 & 1.056 & 5.8 & 24.69 & 27.34 & 1.061 & 7.4 & 44.78 & 49.85 & 1.054 & 6.2 & 40.11 & 43.90 & 1.049 & 6.6 \\
\hline DNZ-Leu & 26.99 & 27.58 & 1.015 & 1.7 & 24.30 & 24.84 & 1.013 & 1.8 & 32.60 & 33.32 & 1.013 & 1.7 & 31.32 & 31.96 & 1.012 & 1.6 \\
\hline DNZ- $\alpha$ MeLeu & 19.60 & - & - & - & 13.43 & 13.53 & 1.005 & $<0.7$ & 24.75 & - & - & - & 21.84 & - & - & - \\
\hline DNZ-NMeLeu & 30.91 & 31.16 & 1.006 & $<0.7$ & 22.59 & 22.69 & 1.003 & $<0.7$ & 32.31 & - & - & - & 28.44 & - & - & - \\
\hline DNZ-Phe & 22.70 & - & - & - & 19.24 & - & - & - & 26.31 & 26.54 & 1.006 & $<0.7$ & 24.51 & 24.77 & 1.007 & 0.7 \\
\hline DNZ- $\beta$ Phe & 39.41 & - & - & - & 27.87 & - & - & - & 52.04 & - & - & - & 49.50 & - & - & - \\
\hline DNZ-Pro & 30.49 & - & - & - & 19.11 & - & - & - & 29.80 & - & - & - & 27.02 & - & - & - \\
\hline DNZ- $\alpha \mathrm{Abu}$ & 24.03 & 24.46 & 1.013 & 1.5 & 20.04 & 20.40 & 1.011 & 1.6 & 30.14 & 30.68 & 1.011 & 1.4 & 25.89 & 26.36 & 1.012 & 1.5 \\
\hline DNZ- $\beta A b u$ & 52.51 & - & - & - & 38.73 & - & - & - & 48.84 & - & - & - & 59.14 & 59.92 & 1.006 & $<0.7$ \\
\hline DNZ-PA & 27.96 & - & - & - & 22.71 & - & - & - & 35.08 & - & - & - & 30.96 & - & - & - \\
\hline Bz-Leu & 25.37 & 26.07 & 1.020 & 2.3 & 20.90 & 21.55 & 1.019 & 2.6 & 28.41 & 29.30 & 1.019 & 1.7 & 25.82 & 26.52 & 1.017 & 2.1 \\
\hline Bz-Phe & 19.84 & 20.05 & 1.008 & $<0.7$ & 19.74 & - & - & - & 22.25 & 22.61 & 1.011 & 1 & 19.11 & 19.39 & 1.010 & 1.2 \\
\hline Bz- $\beta$ Phe & 31.84 & - & - & - & 30.41 & 30.66 & 1.005 & $<0.7$ & 42.47 & 43.42 & 1.012 & 0.9 & 31.69 & 32.12 & 1.008 & 0.9 \\
\hline $\mathrm{Bz}-\alpha \mathrm{Abu}$ & 18.63 & 19.09 & 1.019 & 1.8 & 16.14 & 16.52 & 1.016 & 2 & 22.94 & 23.50 & 1.016 & 1.4 & 20.60 & 21.04 & 1.014 & 1.5 \\
\hline $\mathrm{Bz}-\beta \mathrm{Abu}$ & 39.41 & - & - & - & 36.98 & - & - & $<0.7$ & 54.85 & 56.15 & 1.011 & $<0.7$ & 45.06 & 45.72 & 1.007 & $<0.7$ \\
\hline $\mathrm{Bz}-\mathrm{PA}$ & 23.55 & - & - & - & 27.94 & - & - & - & 30.04 & - & - & - & 25.26 & - & - & - \\
\hline
\end{tabular}

${ }^{\mathrm{a}}$ Conditions as described in Section 2.3.

${ }^{\mathrm{b}}$ Data from Ref. [22].

${ }^{\mathrm{c}}$-: No enantiomeric separation observed $\left(R_{s}<0.5\right)$. 
tration of octanoic acid was varied and the influence of the resulting $\mathrm{pH}_{\mathrm{a}}$ and resolution values was studied (cf. Fig. 4). A significant increase in $R_{s}$ appeared by lowering the $\mathrm{pH}_{\mathrm{a}}$ and an optimum was found at the $100 \mathrm{~m} M$ octanoic acid concentration $\left(R_{s}=56.2\right.$ for DNB-Leu). The highest enantioselectivity was found at the $200 \mathrm{~m} M$ concentration $(\alpha=$ 1.653 for DNB-Leu) but at this higher acid concentration $\mu_{\mathrm{e}}$ and $\mu_{\mathrm{EOF}}$ were further decreased, resulting in higher migration times, lower efficiencies and increasing tendency to peak leading. A 100 $\mathrm{m} M$ concentration of octanoic acid and a $10 \mathrm{mM}$ concentration of chiral counter-ion were found to be suitable for all selectors and solutes investigated. Under the conditions selected, good results with respect to the repeatability of migration and resolution were obtained, as illustrated by the RSD values obtained for DNZ-Phe with tBuCQN as selector $(0.56,0.92$ and $1.59 \%$ for the migration times of the first and the second migrating enantiomer and resolution, respectively).

Using these experimental conditions, the tertiary quinuclidine moiety within the chiral SO (cf. Fig. 1) is protonated and may interact with the negatively charged SAs by ionic interaction to form electrically neutral ion-pairs. Due to their opposite charge, free SO and SAs species exhibit countercurrent-like electrophoretic migration and their overall velocity is also influenced by the $\mu_{\mathrm{EOF}}$ of the system. On the other hand, the neutral ion-pairs will move only with the $\mu_{\mathrm{EOF}}$ (cathodic flow). Thus, the free and complexed SA species show significantly different mobilities, a fact that gives rise to high enantioselectivity. The difference between the ion-pair formation

Table 2

Enantioresolution of amino acid derivatives with tBuCQN, tBuCQD, DNPCQN and cHexCQN as selectors ${ }^{\mathrm{a}}$

\begin{tabular}{|c|c|c|c|c|c|c|c|c|c|c|c|c|c|c|c|c|}
\hline \multirow[t]{2}{*}{ Analyte } & \multicolumn{4}{|c|}{$\mathrm{tBuCQN}$} & \multicolumn{4}{|c|}{ tBuCQD } & \multicolumn{4}{|c|}{ DNPCQN } & \multicolumn{4}{|c|}{ cHexCQN } \\
\hline & $\begin{array}{l}t_{1} \\
(\min )\end{array}$ & $\begin{array}{l}t_{2} \\
(\min )\end{array}$ & $\alpha$ & $R_{s}$ & $\begin{array}{l}t_{1} \\
(\min )\end{array}$ & $\begin{array}{l}t_{2} \\
(\min )\end{array}$ & $\alpha$ & $R_{s}$ & $\begin{array}{l}t_{1} \\
(\mathrm{~min})\end{array}$ & $\begin{array}{l}t_{2} \\
(\min )\end{array}$ & $\alpha$ & $R_{s}$ & $\begin{array}{l}t_{1} \\
(\min )\end{array}$ & $\begin{array}{l}t_{2} \\
(\min )\end{array}$ & $\alpha$ & $R_{s}$ \\
\hline DNB-Leu ${ }^{b}$ & 15.97 & 36.14 & 1.572 & 64.3 & 19.84 & 51.32 & 1.783 & 78.3 & 19.52 & 23.98 & 1.151 & 17.3 & 19.55 & 44.58 & 1.787 & 66.9 \\
\hline DNB-Phg & 15.67 & 26.65 & 1.370 & 48.9 & 15.00 & 30.72 & 1.650 & 66.4 & 15.34 & 17.60 & 1.107 & 10.2 & 14.83 & 28.86 & 1.673 & 59.6 \\
\hline DNB-tLeu & 16.97 & 34.14 & 1.473 & 57.3 & 16.72 & 36.06 & 1.673 & 71.5 & 16.61 & 22.72 & 1.247 & 24.3 & 17.17 & 40.71 & 1.871 & 70.8 \\
\hline DNB- $\alpha$ MeLeu & 12.31 & 12.72 & 1.023 & 2.8 & 14.32 & 15.19 & 1.046 & 5.7 & 14.78 & 15.36 & 1.029 & 3.1 & 13.96 & 14.60 & 1.038 & 4.3 \\
\hline DNB-NMeLeu & 12.95 & 13.11 & 1.008 & $<0.7$ & 15.02 & $-^{\mathrm{c}}$ & - & $<0.7$ & 16.55 & 16.70 & 1.007 & $<0.7$ & 16.39 & 16.54 & 1.007 & $<0.7$ \\
\hline DNB-Phe ${ }^{b}$ & 16.94 & 35.66 & 1.504 & 61.1 & 16.28 & 38.03 & 1.759 & 80 & 18.30 & 22.60 & 1.158 & 13.4 & 16.82 & 40.78 & 1.905 & 56.2 \\
\hline DNB-Pro & 13.06 & - & - & - & 17.77 & - & - & - & 24.93 & - & - & - & 15.51 & - & - & - \\
\hline DNB- $\alpha \mathrm{Abu}$ & 16.86 & 35.50 & 1.506 & 61.7 & 16.07 & 37.29 & 1.757 & 90.8 & 17.18 & 20.39 & 1.130 & 10.1 & 16.57 & 32.61 & 1.663 & 55.4 \\
\hline DNB- $\beta A b u$ & 37.64 & 53.02 & 1.147 & 17.1 & 27.76 & 61.43 & 1.544 & 45.5 & 41.07 & 50.76 & 1.113 & 11.9 & 35.52 & 61.55 & 1.392 & 43.5 \\
\hline DNZ-Leu & 28.80 & 38.95 & 1.153 & 20.6 & 25.94 & 36.22 & 1.230 & 25.5 & 28.04 & 30.95 & 1.063 & 6.8 & 24.30 & 31.62 & 1.209 & 19 \\
\hline DNZ- $\alpha$ MeLeu & 18.95 & 19.19 & 1.008 & 1.1 & 21.36 & 21.65 & 1.010 & 1.1 & 20.59 & 21.27 & 1.023 & 2.8 & 18.95 & 19.32 & 1.016 & 1.8 \\
\hline DNZ-NMeLeu & 26.05 & 26.78 & 1.015 & 1.8 & 25.11 & 25.75 & 1.017 & 1.8 & 40.91 & 41.50 & 1.008 & 0.9 & 24.33 & 24.68 & 1.011 & 0.9 \\
\hline DNZ-Phe & 20.98 & 25.40 & 1.114 & 14.5 & 20.47 & 25.56 & 1.165 & 18.2 & 21.47 & 26.94 & 1.162 & 16.3 & 21.01 & 27.19 & 1.212 & 19 \\
\hline DNZ- $\beta$ Phe & 40.01 & 47.21 & 1.070 & 9.8 & 41.58 & 50.92 & 1.111 & 14.7 & 47.58 & 55.23 & 1.074 & 8.6 & 37.46 & 44.56 & 1.117 & 11.8 \\
\hline DNZ-Pro & 24.55 & 25.52 & 1.021 & 1.4 & 26.21 & 27.29 & 1.027 & 1.6 & 35.22 & 37.13 & 1.030 & 2.4 & 23.45 & 24.11 & 1.021 & 1.1 \\
\hline DNZ- $\alpha \mathrm{Abu}$ & 23.76 & 30.62 & 1.017 & 19.4 & 22.03 & 28.90 & 1.197 & 22.5 & 30.16 & 34.54 & 1.084 & 9.9 & 20.81 & 25.99 & 1.182 & 17.7 \\
\hline DNZ- $\beta A b u$ & 28.10 & 32.95 & 1.082 & 10.1 & 33.79 & 40.84 & 1.115 & 11.8 & 75.06 & 84.12 & 1.043 & 4.2 & 51.65 & 59.46 & 1.082 & 8.8 \\
\hline DNZ-PA & 28.65 & 29.11 & 1.008 & 1.1 & 26.77 & 27.09 & 0.867 & 0.94 & 28.77 & 29.21 & 1.009 & $<0.7$ & 27.57 & - & - & - \\
\hline Bz-Leu & 24.56 & 29.11 & 1.094 & 12.4 & 23.69 & 29.57 & 1.156 & 17.1 & 39.93 & 41.83 & 1.025 & 3.3 & 24.91 & 28.45 & 1.102 & 11 \\
\hline Bz-Phe & 20.85 & 23.51 & 1.072 & 10.1 & 16.92 & 19.55 & 1.112 & 13.3 & 16.94 & 17.77 & 1.036 & 3.5 & 18.57 & 20.76 & 1.091 & 10 \\
\hline Bz- $\beta$ Phe & 37.00 & 40.49 & 1.040 & 5.1 & 28.83 & 31.54 & 1.058 & 6.7 & 31.98 & 32.90 & 1.017 & 1.8 & 30.75 & 32.66 & 1.042 & 4.4 \\
\hline $\mathrm{Bz}-\alpha \mathrm{Abu}$ & 18.95 & 21.46 & 1.077 & 9.6 & 18.26 & 20.77 & 1.097 & 11.2 & 18.94 & 19.57 & 1.024 & 1.4 & 17.90 & 19.29 & 1.061 & 5.5 \\
\hline $\mathrm{Bz}-\beta \mathrm{Abu}$ & 48.32 & 52.36 & 1.030 & 3.5 & 38.93 & 41.46 & 1.036 & 2.3 & 48.86 & 49.88 & 1.010 & $<0.7$ & 44.86 & - & - & - \\
\hline $\mathrm{Bz}-\mathrm{PA}$ & 17.25 & - & - & - & 19.51 & - & - & - & 18.09 & 18.39 & 1.012 & $<0.7$ & 22.62 & - & - & - \\
\hline
\end{tabular}

\footnotetext{
${ }^{\text {a }}$ Conditions as described in Section 2.3 .

${ }^{\mathrm{b}}$ Data from Ref. [22].

${ }^{\mathrm{c}}$-: No enantiomeric separation observed $\left(R_{s}<0.5\right)$.
} 
constants for $(R)$ - and $(S)$-enantiomers of the SAs, based on the enantioselective intermolecular interactions mentioned above, represents the other contribution to selectivity in these systems.

\subsection{Cinchona alkaloids as chiral selectors}

The enantioresolution of all amino acid derivatives was studied first with the four natural cinchona alkaloids under the selected operating conditions.

Using QN, QD, CN and CD, the migration times $(t)$, the enantioselectivity $(\alpha)$ and the resolution $\left(R_{s}\right)$ for the two SAs enantiomers are presented in Table 1 .

Rather poor enantioseparations were observed for the amino acid derivatives examined using these SOs. Compared to $\mathrm{DNZ}$ and $\mathrm{Bz}$ derivatives, the DNB derivatives seem, however, to be favourable with respect to enantiodiscrimination capability of these types of SOs. Using QN and CD, the $(S)$-DNBLeu enantiomer migrated first but with QD and $\mathrm{CN}$, the $(R)$-DNB-Leu enantiomer migrated first. Higher mobility differences and selectivity values were obtained for DNB derivatives enantiomers using QD and, compared to $\mathrm{QN}$, enantiomeric separations could be achieved for DNZ- $\alpha$ MeLeu, Bz- $\beta$ Phe and $\mathrm{Bz}-\beta \mathrm{Abu}$. The following order in $\alpha$ values is observed: $\mathrm{QD}>\mathrm{QN}>\mathrm{CN}>\mathrm{CD}$.

\subsection{Quinine and quinidine carbamoylated derivatives as chiral selectors}

Using tBuCQN, tBuCQD, DNPCQN and cHexCQN, $t, \alpha$ and $R_{s}$ are presented in Table 2.

In most cases higher resolution values were obtained with these SOs, resulting in the enantioseparation of all the derivatives excepted DNB-Pro and Bz-PA (which is partly separated using DNPCQN). The additional substituent (tert.-butyl, dinitrophenyl or cyclohexyl) in the SO and in particular the carbamate function, that may serve as hydrogen donor-acceptor, has obviously a favourable effect on enantioselectivity. It is also worth noting that in the presence of carbamoylated QN derivatives the migration order was reversed compared to QN: the $(R)$ DNB-Leu enantiomer migrated before the $(S)$-DNBLeu enantiomer with tBuCQN, DNPCQN and cHexCQN. This reversal of migration order, which has also been observed in the corresponding liquid chromatographic enantioseparations with chiral

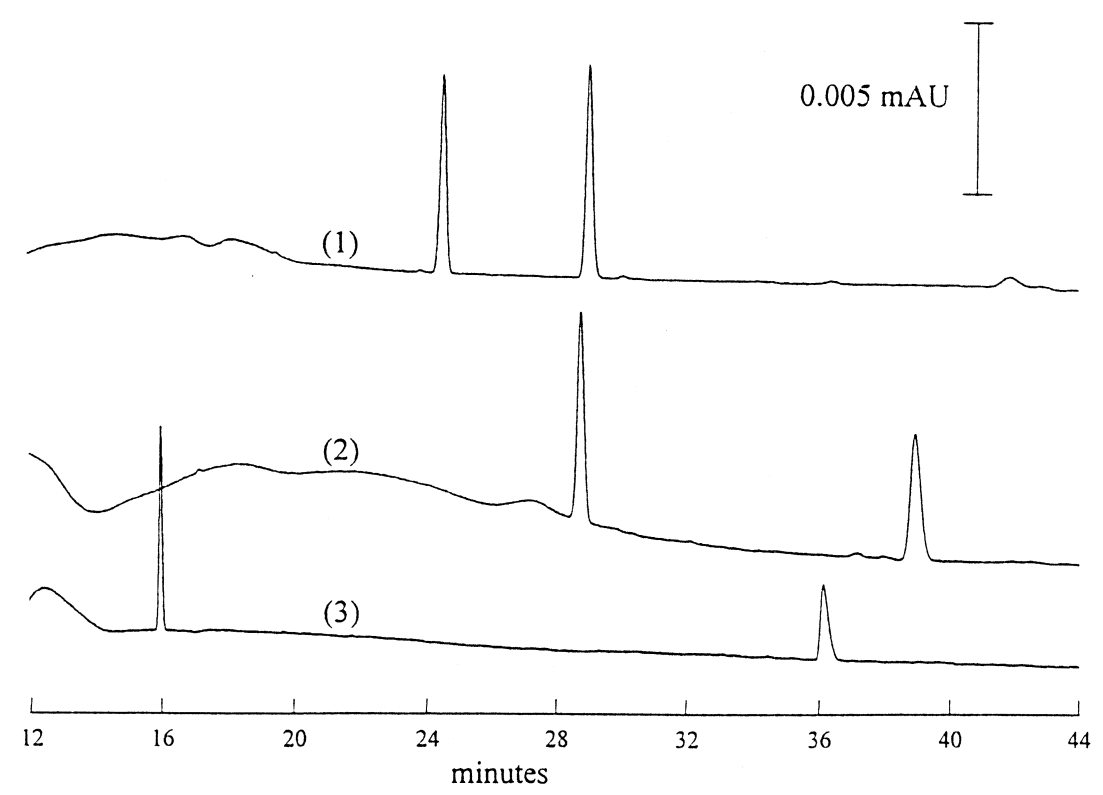

Fig. 5. Enantioseparation of (1) Bz-, (2) DNZ- and (3) DNB-Leu. Buffer: $100 \mathrm{~m} M$ octanoic acid and $12.5 \mathrm{~m} M$ ammonia in methanol-ethanol (40:60) containing $10 \mathrm{~m} M$ tBuCQN. 
stationary phases based on QN and carbamoylated QN derivatives as chiral SOs, is probably due to a change of relative binding affinities of the corresponding SA enantiomers and indicates a change in the chiral recognition mechanism. Using tBuCQD, the $(S)$ - migrates before the $(R)$-DNB-Leu enantiomer $\left[R_{s}=78.3, \alpha=1.783, N(S)=129000, A_{\mathrm{s}}(S)=\right.$ 0.96] and the enantioselectivities were higher than using tBuCQN [for DNB-Leu, $R_{s}=64.3, \alpha=1.572$, $\left.N(S)=127000, A_{\mathrm{s}}(S)=0.93\right]$. With DNPCQN, lower enantioseparations were observed but Bz-PA enantiomers could be separated. CHexCQN gave selectivities almost as high as tBuCQD. The following order in $\alpha$ values is observed: cHexCQN $>$ tBuCQD $>$ tBuCQN $>$ DNPCQN.

For example, Fig. 5 presents the electropherograms obtained for Bz-Leu, DNZ-Leu and DNB-Leu with tBuCQN as SO.

\section{Conclusions}

A NACE system using a background electrolyte made of $100 \mathrm{~m} M$ octanoic acid and $12.5 \mathrm{~m} M$ ammonia in ethanol-methanol (60:40) was applied to the investigation of the potential of $\mathrm{QN}$ chiral derivatives for the enantioseparation of $N$-protected amino acids. The best enantioseparations were obtained using DNB derivatives as SAs and carbamoylated derivatives as SOs $(\alpha=1.905$ for DNBPhe using cHexCQN). In further work, the collected enantioselectivity values will be correlated with those obtained in HPLC using the same SOs immobilized onto silica as chiral stationary phase in order to apply this NACE method as a screening tool for a fast evaluation of the chiral discrimination potential of a larger set of newly developed quinine and quinidine derivatives.

\section{References}

[1] G. Gübitz, M. Schmid, J. Chromatogr. A 792 (1997) 179_ 225.

[2] R. Vespalec, P. Bocek, Electrophoresis 18 (1997) 843-852.

[3] H.J. Issaq, K.C. Chan, Electrophoresis 16 (1995) 467-480.

[4] B. Chankvetadze, Capillary Electrophoresis in Chiral Analysis, Wiley, Chichester, 1997.

[5] A.M. Stalcup, K.H. Gahm, J. Microcol. Sep. 8 (1996) 145150.

[6] I. Bjornsdottir, S.H. Hansen, S. Terabe, J. Chromatogr. A 745 (1996) 37-44.

[7] C. Rosini, G. Uccello-Barretta, D. Pini, C. Abete, P. Salvadori, J. Org. Chem. 53 (1988) 4579-4581.

[8] C. Pettersson, J. Chromatogr. A 316 (1984) 553-567.

[9] C. Pettersson, G. Schill, J. Liq. Chromatogr. 9 (1986) 269290.

[10] M. Lämmerhofer, W. Lindner, J. Chromatogr. A 829 (1998) $115-123$

[11] C. Rosini, P. Altemura, D. Pini, C. Bertucci, G. Zullino, P. Salvadori, J. Chromatogr. A 348 (1985) 79-87.

[12] P. Salvadori, C. Rosini, D. Pini, C. Bertucci, G. UccelloBarretta, Chirality 1 (1989) 161-169.

[13] M. Lämmerhofer, W. Lindner, GIT Special: Chromatogr. Int. 40 (No. 6) (1996) 16-20.

[14] M. Lämmerhofer, W. Lindner, J. Chromatogr. A 741 (1996) 33-48.

[15] M. Lämmerhofer, N.M. Maier, W. Lindner, Am. Lab. 30 (1998) 71-78.

[16] M. Lämmerhofer, P. Di Eugenio, I. Molnar, W. Lindner, J. Chromatogr. B 689 (1997) 123-135.

[17] O.P. Kleidernigg, M. Lämmerhofer, W. Lindner, Enantiomer 1 (1996) 387-391.

[18] V. Piette, M. Lämmerhofer, K. Bischoff, W. Lindner, Chirality 9 (1997) $157-161$.

[19] V. Piette, M. Lämmerhofer, W. Lindner, J. Crommen, Chirality 11 (1999) 622-630.

[20] W. Lindner, M. Lämmerhofer, N.M. Maier, PCT/EP97/ 02888, 1997.

[21] The European Pharmacopoeia, 3rd ed., Council of Europe, Strasbourg, 1996, Part 2.2.2.

[22] V. Piette, M. Fillet, W. Lindner, J. Crommen, Biomed. Chromatogr. (1999), in press. 